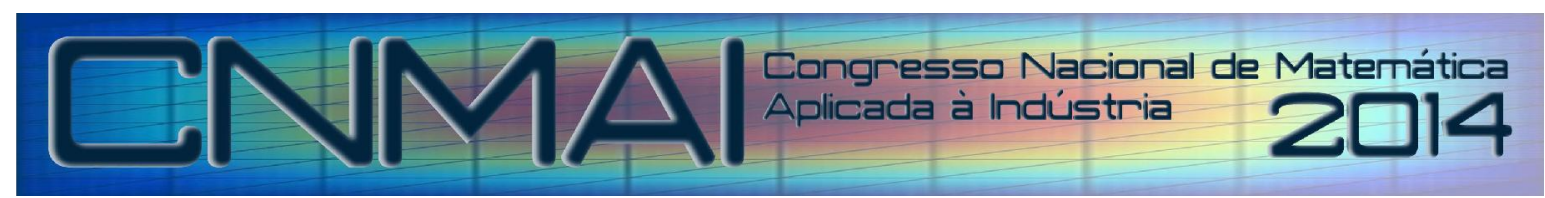

18 a 21 de novembro de 2014, Caldas Novas - Goiás

\title{
OTIMIZAÇÃO DE CIRCUITOS MULTIMODAIS PARA CONTROLE PASSIVO DE VIBRAÇÕES DE ESTRUTURAS COMPOSTAS
}

\author{
Victor Augusto da Costa Silva, Victor_augusto_cn@hotmail.com \\ Bruno Gabriel Gustavo Leonardo Zambolini Vicente, Brunozv@gmail.com \\ Antônio Marcos Gonçalves de Lima, amglima@mecanica.ufu.br
}

Universidade Federal de Uberlândia - Faculdade de Engenharia Mecânica, Campus Santa Mônica, Uberlândia-MG

Universidade Federal de Uberlândia - Faculdade de Engenharia Mecânica, Campus Santa Mônica, Uberlândia-MG

Universidade Federal de Uberlândia - Faculdade de Engenharia Mecânica, Campus Santa Mônica, Uberlândia-MG

Resumo: Nas ultimas décadas, muitos esforços tem sido direcionados à modelagem e projeto de sistemas de engenharia incorporando estruturas inteligentes para o problema de atenuar a vibração através da combinação de avanços tecnológicos nas áreas de ciência dos materiais e comutação, e de técnicas numéricas de otimização. Entre os materiais inteligentes mais utilizados, pode citar-se o uso de materiais piezoelétricos a serem combinados com circuitos elétricos passivos. Entretanto, a estratégia mais comum que tem sido empregada é o uso de circuitos shunt que controlam apenas um modo de vibração. Assim, para controlar mais modos de vibração de uma estrutura de engenharia, vários elementos piezoelétricos incorporados na estrutura são necessários, onde cada elemento piezoelétrico está associado a um circuito de um modo de vibração específico, causando certos problemas à estrutura como acréscimo de massa causado pelas pastilhas piezoelétricas. Por outro lado, circuitos elétricos passivos multimodais que controlam vários modos de vibração simultaneamente utilizando apenas um elemento piezoelétrico apresenta vantagens substanciais. $O$ projeto efetivo de circuitos shunt multimodais aplicados à estruturas de engenharia complexas pode ser convenientemente obtido utilizando técnicas de otimização numérica multiobjetiva robusta que são capazes de lhe dar com vários objetivos e funções vulnerabilidade, algumas delas podendo ser conflitantes entre si. Porém, o número grande de cálculos das funções vulnerabilidade, combinado com modelos de elementos finitos de grandes dimensões de estruturas industriais, tornam a otimização robusta muito cara computacionalmente, às vezes impossíveis de se realizar. Estas dificuldades motivam o estudo mostrado neste trabalho, em que uma estratégia é proposta consistindo no uso de algoritmos evolucionários multiobjetivos combinados com metamodelos. Após a discussão de alguns aspectos teóricos envolvendo neste estudo, uma aplicação numérica é apresentada para ilustrar a metodologia de otimização multiobjetivo robusta para o projeto ótimo-robusto de circuitos shunt multimodais aplicados em placas compostas.

Palavras-chave: Otimização Robusta, Estruturas Compostas, Circuitos Shunt Multimodais, Controle Passivo, Incertezas

\section{INTRODUÇÃO}

Como resultado dos avanços tecnológicos na ciência dos materiais e no desenvolvimento atual de técnicas de controle e otimização de estruturas, existe um aumento no uso das chamadas Estruturas Inteligentes. Estas estruturas podem identificar mudanças nas condições ambientais e/ou estruturais e realizar uma auto adaptação com o objetivo de assegurar desempenho satisfatório. Neste sentido, a implementação de estruturas inteligentes se estende para setores onde é intrínseco maximizar a segurança, confiabilidade e desempenho, como o desenvolvimento de estruturas espaciais, satélites de comunicação, setor automotivo, de construção, equipamentos esportivos, entre outros (Faria, 2006).

Dentre os materiais inteligentes mais utilizados, as cerâmicas piezelétricas são consideradas interessantes em muitos tipos de aplicação. Por outro lado, os chamados materiais compostos se mostram como uma possibilidade atraente no projeto de estruturas inteligentes, pelo fato de possuírem um comportamento não convencional apropriado a necessidades específicas de sua aplicação. 
Uma possibilidade interessante no uso dos materiais piezelétricos é o controle passivo de vibrações e ruídos, aplicando este material em virtude deles possibilitarem a conversão de parte da deformação da estrutura em energia elétrica durante o ciclo de vibração. Esta energia pode ser dissipada ou transformada através de um circuito passivo (circuito shunt) constituindo uma forma de controle passivo de estruturas (Viana, 2005).

O modelo mais comum é o circuito shunt que controla um único modo, comumente conhecido como circuito elétrico shunt monomodal (Viana, 2006; Hagood \& Flotow, 1991; Wu, 1996). Este pode ser resistivo ou ressonante, em série ou em paralelo. Entretanto, para o controle de mais modos de vibração de uma estrutura, são necessários vários elementos piezelétricos acoplados na estrutura, cada PZT relacionado a um circuito de um modo específico de vibração (Viana, 2006). Porém, isto causa certos problemas, pois a estrutura pode não possuir espaço suficiente para acomodar uma grande quantidade de PZTs, além da modificação das propriedades estruturais pela mudança em sua rigidez e o aumento excessivo de sua massa. Com isso, surge a necessidade de um circuito shunt que controle vários modos simultaneamente e usando apenas um PZT. Duas topologias de circuitos elétricos shunt multimodais são utilizados neste trabalho: circuito ressonante em paralelo proposto por Wu (1998), e o ressonante em série proposto por Moheimani e Fleming (2003).

Apesar da existência de várias formulações analíticas para sintonização ótima de circuitos shunt de topologias mais simples, como o especificado em Hagood e Von Flotow (1991), estes parâmetros, normalmente, não são tão óbvios em topologias mais complexas. Além disso, pequenas variações nos parâmetros do circuito levam à perda de sua eficiência. Com isso, são necessárias, neste caso, técnicas numéricas para otimização multiobjetivo levando em consideração incertezas inerentes nos parâmetros do circuito para obter projetos mais robustos. Neste trabalho, a aproximação a ser utilizada para a criação do modelo probabilístico de um circuito elétrico shunt será o Princípio da Máxima Entropia, que é baseado na teoria da informação, que a partir das funções densidade de probabilidade de cada variável, os valores destas variáveis incertas são aleatoriamente selecionados através do uso de um solver estocástico.

Neste caso, uma variante do método de Monte Carlo, normalmente conhecida como Hyper - Cubo - Latino (HCL), com convergência garantida para pequenas amostras, será utilizada. Devido ao grande esforço computacional no cálculo das funções vulnerabilidade associadas às funções objetivo, foi necessária a combinação da otimização robusta com a metamodelagem, utilizando redes neurais.

O interesse é a construção dos envelopes das funções de resposta em frequência que determinam os intervalos de confiança de cada variável na resposta do sistema contendo o circuito shunt.

\section{MODELAGEM POR ELEMENTOS FINITOS}

Neste trabalho, será utilizada a Teoria Mista, combinando a Teoria da Camada Equivalente Única na modelagem da parte mecânica da estrutura, com a discretização dos potenciais elétricos sendo feito por camadas, utilizando a Teoria da Camada Equivalente Discreta. Os potenciais elétricos são considerados contínuos em cada camada, apresentando uma variação linear ao longo da espessura. O elemento finito considerado neste trabalho é da família Serendipity, composto por oito nós com funções de forma polinomiais. A Teoria de Deformação Cisalhante de Primeira Ordem (FSDT), composta por 5 graus de liberdade por nó, sendo recomendada para modelagem de placas finas, possui os seguintes campos de deslocamento (Reddy, 1997):

$$
\begin{aligned}
& u(x, y, z, t)=u_{0}(x, y, t)+z \psi_{x}(x, y, t) \\
& v(x, y, z, t)=v_{0}(x, y, t)+z \psi_{y}(x, y, t) \\
& w(x, y, z, t)=w_{0}(x, y, t)
\end{aligned}
$$

onde $u_{0}, v_{0}, w_{0}, \psi_{x} \mathrm{e} \psi_{y}$ são os graus de liberdade. A partir das relações entre deslocamentos e deformações, é possível obter a seguinte relação:

$$
\boldsymbol{\varepsilon}(\xi, \eta, z, t)=\mathbf{D}(z) \mathbf{N}_{u}(\xi, \eta) \mathbf{u}_{e}(t)
$$

onde $\boldsymbol{D}(z)$ é a matriz de operadores diferenciais, e $\boldsymbol{N}_{u}$ é matriz das funções de forma. Assim, a partir das energias cinética e potencial, as matrizes de massa e rigidez mecânica são expressas como:

$$
\begin{gathered}
\mathbf{M}_{e}=\int_{V_{e}} \rho \mathbf{N}^{T} \mathbf{A}^{T} \mathbf{A} \mathbf{N} d V_{e} \\
\mathbf{K}_{u u}^{e}=\sum_{k=1}^{n c} \int_{\xi=-1}^{+1} \int_{\eta=-1}^{+1} \int_{z=z_{k}}^{z=z_{k+1}} \mathbf{B}_{u}{ }^{T} \mathbf{T}^{T} \mathbf{C} \mathbf{T} \mathbf{B}_{u} J d z d \eta d \xi
\end{gathered}
$$


onde $\boldsymbol{C}$ é a matriz das propriedades mecânicas do material composto, $\boldsymbol{T}$ é a matriz de transformação por rotação, e $\mathbf{B}_{u}=\mathbf{D}(z) \mathbf{N}_{u}$. De acordo com Chee (2000), o potencial elétrico de cada camada é obtido como:

$$
\phi_{\text {camada }(i)}(x, y, z, t)=L_{i d}(z) \phi_{i}(x, y, t)+L_{i u}(z) \phi_{i+1}(x, y, t)
$$

onde $L_{i d}$ e $L_{i u}$ são funções de interpolação Lagrangeanas das interfaces de uma camada $i$ do composto. Com isso, combinando a equação anterior com a definição dada por Hwang e Park (1993), onde o campo elétrico é igual ao gradiente negativo do potencial elétrico, obtém-se:

$$
\left\{\begin{array}{l}
E_{x}(x, y, z, t) \\
E_{y}(x, y, z, t) \\
E_{z}(x, y, z, t)
\end{array}\right\}_{\operatorname{camada}(i)}=-\left\{\begin{array}{c}
L_{i d}(z) \frac{\partial \phi_{i}(x, y, t)}{\partial x}+L_{i u}(z) \frac{\partial \phi_{i+1}(x, y, t)}{\partial x} \\
L_{i d}(z) \frac{\partial \phi_{i}(x, y, t)}{\partial y}+L_{i u}(z) \frac{\partial \phi_{i+1}(x, y, t)}{\partial y} \\
\frac{1}{z_{i}-z_{i+1}} \phi_{i}(x, y, t)+\frac{1}{z_{i+1}-z_{i}} \phi_{i+1}(x, y, t)
\end{array}\right\}
$$

Assim, levando em conta as equações da energia potencial, a relação entre o tensor de tensão e o deslocamento elétrico, e após realizar algumas manipulações matemáticas, as seguintes expressões para as matrizes elementares de rigidez eletromecânica e elétrica são obtidas:

$$
\begin{aligned}
& \mathbf{K}_{u \varphi}^{e}=\sum_{k=1}^{n c} \int_{\xi=-1}^{+1} \int_{\eta=-1}^{+1} \int_{z=z_{k}}^{z=z_{k+1}} \mathbf{B}_{u}{ }^{T} \mathbf{T}^{T} \mathbf{e Q B} \mathbf{B}_{\varphi} J d z d \eta d \xi \\
& \mathbf{K}_{\varphi u}^{e}=\sum_{k=1}^{n c} \int_{\xi=-1}^{+1} \int_{\eta=-1}^{+1} \int_{z=z_{k}}^{z=z_{k+1}} \mathbf{B}_{\varphi}{ }^{T} \mathbf{Q} \mathbf{e T}^{T} \mathbf{B}_{u} J d z d \eta d \xi \\
& \mathbf{K}_{\varphi \varphi}^{e}=\sum_{k=1}^{n c} \int_{\xi=-1}^{+1} \int_{\eta=-1}^{+1} \int_{z=z_{k}}^{z=z_{k+1}} \mathbf{B}_{\varphi}{ }^{T} \mathbf{Q} \chi \mathbf{Q}^{-1} \mathbf{B}_{\varphi} J d z d \eta d \xi
\end{aligned}
$$

onde $\boldsymbol{e}$ é o tensor de constantes dielétricas, $\chi$ é a matriz de permissividade, $\boldsymbol{Q}$ é uma matriz de transformação de coordenadas por rotação, e $\mathbf{B}_{\varphi}=\left[\begin{array}{lll}\partial / \partial \xi & \partial / \partial \eta & \partial / \partial z\end{array}\right]^{T} \mathbf{N}_{\varphi}$.

Através das matrizes de massa e rigidez obtidas anteriormente, e após o procedimento de montagem dos elementos finitos, levando em conta a conectividade dos nós, as equações do movimento a nível global do sistema são geradas:

$$
\left[\begin{array}{cc}
\mathbf{M}_{u u} & 0 \\
0 & 0
\end{array}\right]\left\{\begin{array}{c}
\ddot{\mathbf{u}}_{g} \\
\ddot{\varphi}_{g}
\end{array}\right\}+\left[\begin{array}{cc}
\mathbf{K}_{u u} & \mathbf{K}_{u \varphi} \\
\mathbf{K}_{\varphi u} & \mathbf{K}_{\varphi \varphi}
\end{array}\right]\left\{\begin{array}{c}
\mathbf{u}_{g} \\
\varphi_{g}
\end{array}\right\}=\left\{\begin{array}{c}
\mathbf{F}_{g} \\
\mathbf{Q}_{g}
\end{array}\right\}
$$

A Equação (8) pode ser expressa no domínio de Fourier, sendo que $\overline{\mathbf{Q}}(\omega)=\frac{1}{j \omega} \mathbf{Z}^{-1}(\omega) \mathbf{L} \overline{\boldsymbol{\varphi}}(\omega)$, onde $\boldsymbol{Z}$ é a impedância do circuito e $\boldsymbol{L}$ indica a matriz que seleciona dentre os graus de liberdade, aqueles aos quais os circuitos shunt estão conectados. Com isso, a função de resposta em frequência é expressa como:

$$
\mathbf{H}(\omega)=\left[\mathbf{K}_{u u}-\overline{\mathbf{K}}_{u \varphi}\left(\overline{\mathbf{K}}_{\varphi \varphi}-\frac{\mathbf{Z}^{-1}(\omega)}{j \omega}\right)^{-1} \overline{\mathbf{K}}_{\varphi u}-\omega^{2} \mathbf{M}_{u u}\right]^{-1}
$$

\section{CIRCUITOS SHUNT MULTIMODAIS}

Em 1998, Wu propôs um circuito onde os circuitos de controle seriam do tipo ressonante em paralelo, e cada ramo teria circuitos de bloqueio para que os circuitos de controle dos modos de frequências mais altas não interferissem. Estes circuitos de bloqueio são compostos por um capacitor em paralelo com um indutor, e seus valores são obtidos utilizando a relação $\bar{L}_{i} \bar{C}_{i}=1 / \omega_{i}^{2}$, onde $\omega_{i}$ é a frequência do circuito a ser bloqueado. Um exemplo deste circuito é mostrado na Fig. 1. Os parâmetros dos circuitos de controle são inicialmente calculados como circuitos monomodais (Viana, 2005) e, após isto, os parâmetros dos circuitos multimodais são obtidos (Wu (1998)). 


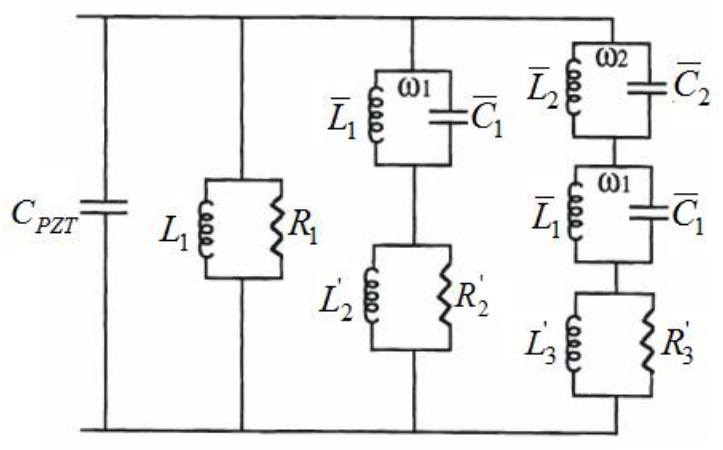

Figura 1. Exemplo de circuito multimodal para três modos (adaptado de Wu (1998)).

Em 2003, Moheimani e Fleming propuseram um circuito similar, com a única diferença sendo os circuitos de controle do tipo ressonante em série. O procedimento para obter os parâmetros do circuito é similar, com as mesmas equações do circuito shunt multimodal utilizadas na outra topologia, para transformar os parâmetros dos circuitos a partir dos parâmetros monomodais. Um exemplo deste tipo de circuito é mostrado na Fig. 2.

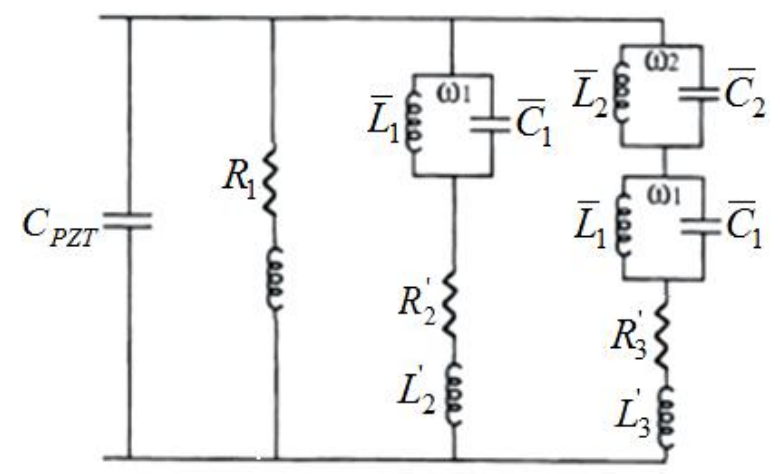

Figura 2. Exemplo de circuito multimodal para três modos proposto por Moheimani e Fleming (2003).

\section{OTIMIZAÇÃO ROBUSTA}

O projeto ótimo de uma estrutura contendo materiais piezelétricos é de grande importância tanto para o ambiente acadêmico como para o ambiente industrial, visando obter projetos ótimos para reduzir custos de construção, aumentar a confiabilidade, robustez e melhor desempenho operacional. Com isso, a otimização é uma ferramenta fundamental para o pré-projeto e/ou projeto inicial, intervindo na modelagem. Além disso, deve-se destacar que a maioria dos problemas práticos de engenharia é de natureza multiobjetivo, com funções custo conflitantes entre si. Então é muito importante escolher uma estratégia de otimização que seja capaz de propor melhores alternativas de projeto. Finalmente, deve-se também levar em consideração possíveis incertezas inerentes nas variáveis de projeto que influem significativamente nas próprias funções custo.

Neste trabalho, incertezas são consideradas na resistência $\left(R_{i}\right)$ e na indutância $\left(L_{i}\right)$ na parte dos circuitos shunt responsáveis pelo controle.

Com o objetivo de avaliar a robustez de uma solução, é aplicada uma função que permite avaliar o impacto das variações dos parâmetros na solução, sendo que esta função é chamada função robustez $f^{r}$, definida como a relação entre a média e o desvio padrão, definida como:

$$
f^{r}=\frac{\mu_{f}}{\sigma_{f}}
$$

onde $\left(\sigma_{f} / \mu_{f}\right)$ é a medida de dispersão, ou a vulnerabilidade de uma função $f(x)$, denotada por $f^{v}(x)$.

O uso de algoritmos genéticos para resolver problemas de otimização multiobjetivo robusta de dinâmica estrutural é muito caro em termos de custo computacional para obter soluções. Assim, é necessário introduzir métodos que aproximem as funções vulnerabilidade com o objetivo de reduzir o custo computacional. Neste trabalho, foram utilizadas redes neurais, mas especificamente o Perceptron Multicamadas. 
Inicialmente, as redes neurais foram treinadas, utilizando as funções objetivo originais. Durante o processo de otimização, foi utilizada a função original para avaliar a primeira função objetivo, e a rede neural para avaliar as funções vulnerabilidade. Utilizando as redes neurais, o tempo computacional gasto foi de cerca de $2 \%$ do tempo que seria gasto utilizando apenas a função original.

\section{RESULTADOS NUMÉRICOS}

Nesta aplicação, será considerada uma estrutura de viga de quatro camadas como mostra a Fig. 3, incorporando um elemento PZT $\left(\rho=7700 \mathrm{~kg} / \mathrm{m}^{3}\right)$ como mostra a mesma figura, que será combinada com um circuito proposto por Wu (1998) e com o circuito desenvolvido por Moheimani e Fleming (2003). O interesse aqui é avaliar o desempenho, a robustez, e as limitações de cada circuito shunt multimodal. A placa composta foi discretizada em seis elementos finitos em que as características geométricas estão definidas na Tab. 1 e mostradas na Fig. 3, onde as dimensões estão em milímetros:

Tabela 1. Características da Placa Composta

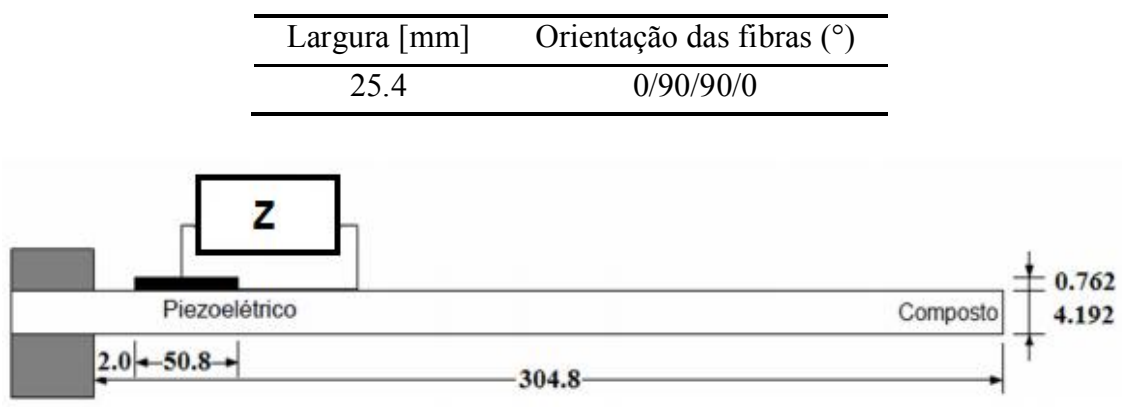

Figura 3. Placa Composta com circuito multimodal.

Dois problemas de otimização são definidos para obter os parâmetros $\mathrm{R}$ e L de cada modo de vibração considerado de interesse, e cada configuração de circuito: (a) determinística, onde a função objetivo original está relacionada com minimizar as amplitudes de vibração dos modos de interesse; (b) robusta, em que a função vulnerabilidade associada à função custo original e relacionada com as incertezas paramétricas introduzidas nas variáveis de projeto, é otimizada em conjunto com a função custo. O procedimento de otimização foi feito com circuitos ressonantes monomodais, pois os resultados obtidos foram melhores do que os obtidos pela otimização do circuito shunt multimodal completo. Em todos os casos, o capacitor de cada circuito de bloqueio é estabelecido com valor de $47 \mathrm{nF}$, e a indutância calculada com a relação mostrada anteriormente. Os circuitos foram acertados para controlar $1^{\circ}, 3^{\circ}$ e $5^{\circ}$ modos.

Os resultados dos dois procedimentos de otimização são mostrados na Tab. 2.

Tabela 2. Resultados dos dois problemas de otimização

\begin{tabular}{|c|c|c|c|c|}
\hline \multirow{2}{*}{ Variáveis } & \multicolumn{2}{|c|}{$\begin{array}{c}\text { Ressonante em } \\
\text { Paralelo }\end{array}$} & \multicolumn{2}{c|}{$\begin{array}{c}\text { Ressonante em } \\
\text { Série }\end{array}$} \\
\cline { 2 - 5 } & Determinística & Robusta & Determinística & Robusta \\
\hline $\mathrm{R}_{1}[\Omega]$ & $2,146 \times 10^{6}$ & $1,745 \times 10^{6}$ & 32270 & 27716 \\
\hline $\mathrm{L}_{1}[\mathrm{H}]$ & 629,6 & 631,42 & 629,1 & 629,45 \\
\hline $\mathrm{R}_{2}[\Omega]$ & $3,539 \times 10^{5}$ & $1,958 \times 10^{5}$ & 6377,4 & 2837,2 \\
\hline $\mathrm{L}_{2}[\mathrm{H}]$ & 17,2 & 17,388 & 19,954 & 17,718 \\
\hline $\mathrm{R}_{3}[\Omega]$ & $1,515 \times 10^{5}$ & $1,111 \times 10^{5}$ & 3987,2 & 16836 \\
\hline $\mathrm{L}_{3}[\mathrm{H}]$ & 3,159 & 3,1822 & 4,5337 & 3,2675 \\
\hline
\end{tabular}

Primeiramente, uma comparação é realizada entre o desempenho dos circuitos shunt multimodais com circuito de controle em paralelo obtidos em ambos os métodos de otimização. As amplitudes da função de resposta em frequência para circuito aberto, circuitos determinístico e robusto são mostradas nas Figs. 4 a 6. 


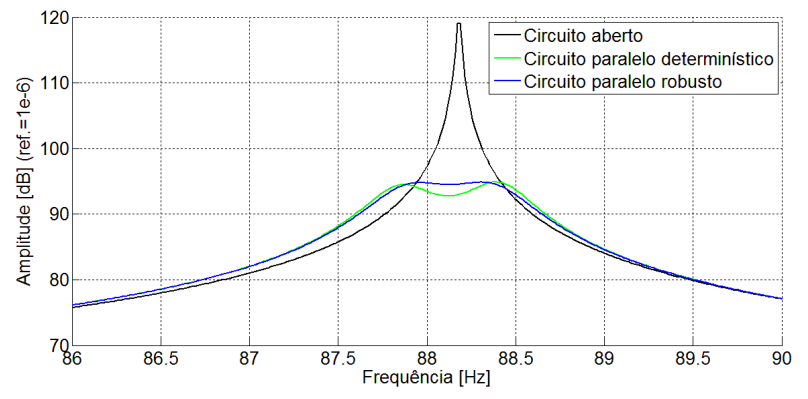

Figura 4. Amplitudes das FRFs para o primeiro modo: Circuito paralelo.

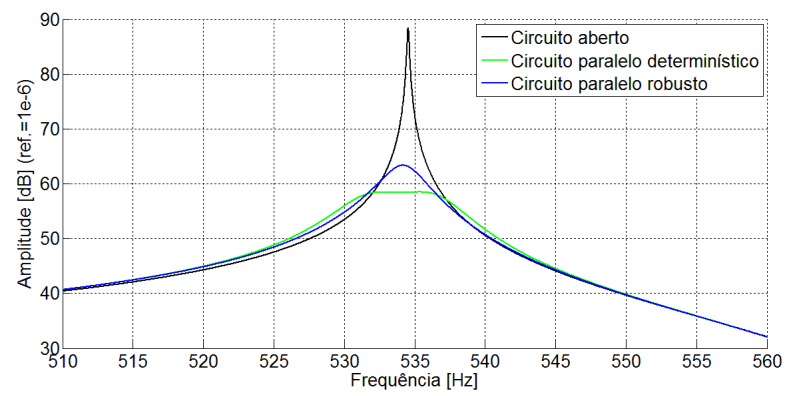

Figura 5. Amplitudes das FRFs para o segundo modo: Circuito paralelo.

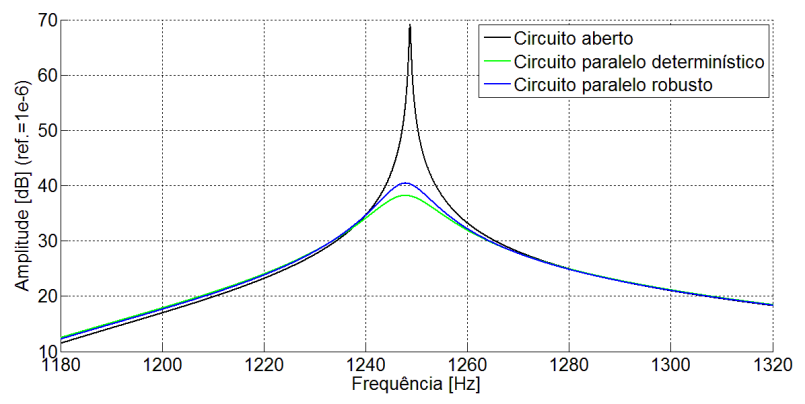

Figura 6. Amplitudes das FRFs para o terceiro modo: Circuito paralelo.

Neste momento, para analisar a estabilidade das soluções robustas comparadas às soluções determinísticas, para cada conjunto de soluções, são geradas aleatoriamente 2000 amostras de pontos utilizando o chamado Hyper-CuboLatino (HCL), assumindo uma função de distribuição Gamma, de acordo com Zambolini-Vicente (2013). O interesse é calcular os envelopes de soluções em termos das amplitudes das FRFs e determinar as propriedades estatísticas em termos de máximos, médios e mínimos valores para cada ponto de frequência. Para gerar as amostras, foram considerados os seguintes níveis de dispersão das variáveis de projeto: $\Delta \mathrm{Ri}=5.0 \%$, e $\Delta \mathrm{Li}=5.0 \%$, com i $=1, \ldots, 3$. Os envelopes de soluções são mostrados nas Figs. 7 a 9:
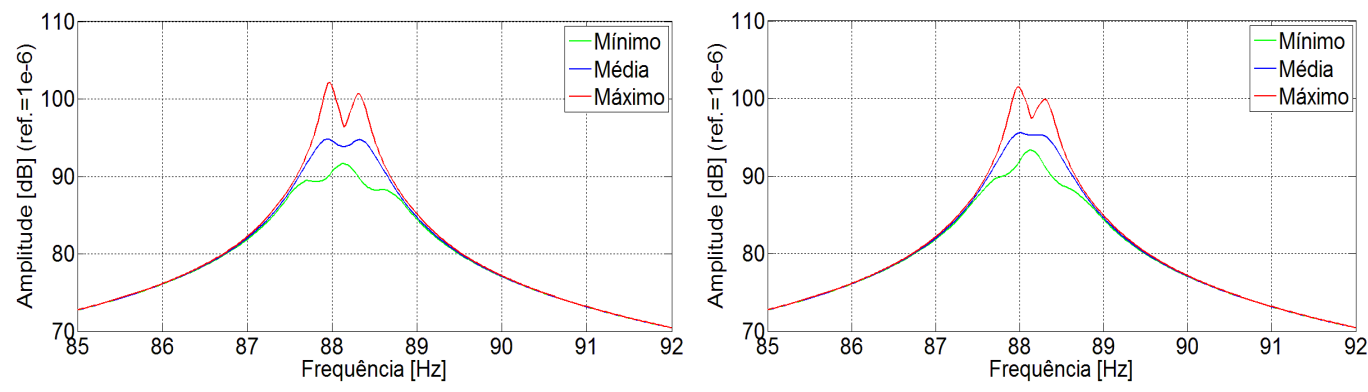

Figura 7. Envelopes de soluções para o primeiro modo - (a) Determinístico, (b) Robusto - Circuito Paralelo. 

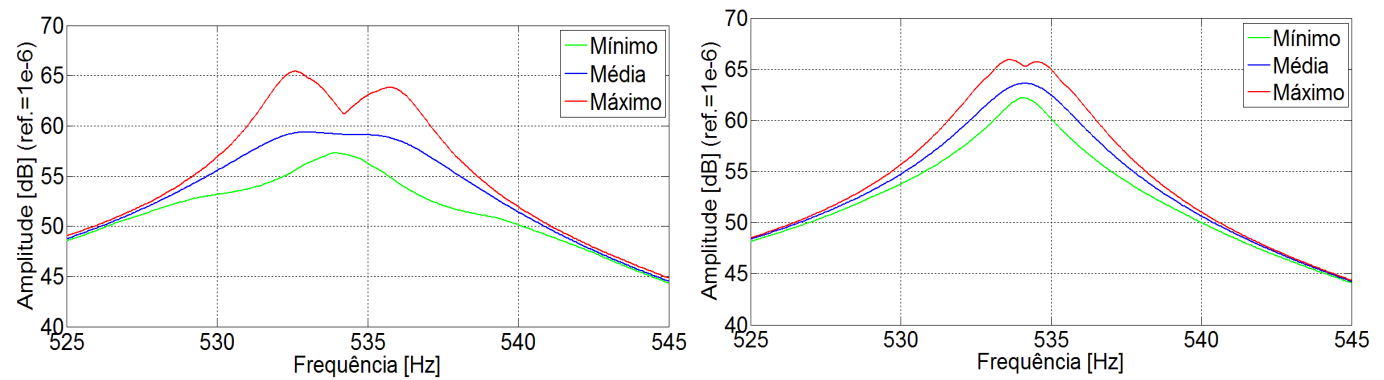

Figura 8. Envelopes de soluções para o segundo modo - (a) Determinístico, (b) Robusto - Circuito Paralelo.
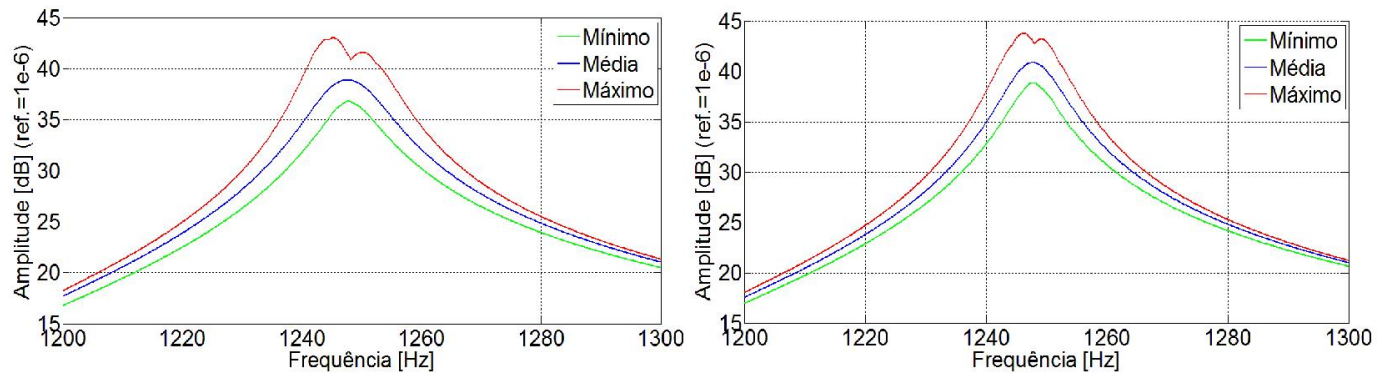

Figura 9. Envelopes de soluções para o terceiro modo - (a) Determinístico, (b) Robusto - Circuito Paralelo.

O mesmo processo foi realizado para o circuito proposto por Moheimani e Flaming (2003). As FRFs obtidas são mostradas nas Figs. 10 a 12, e os envelopes de FRFs são mostrados nas Figs. 13 a 15.

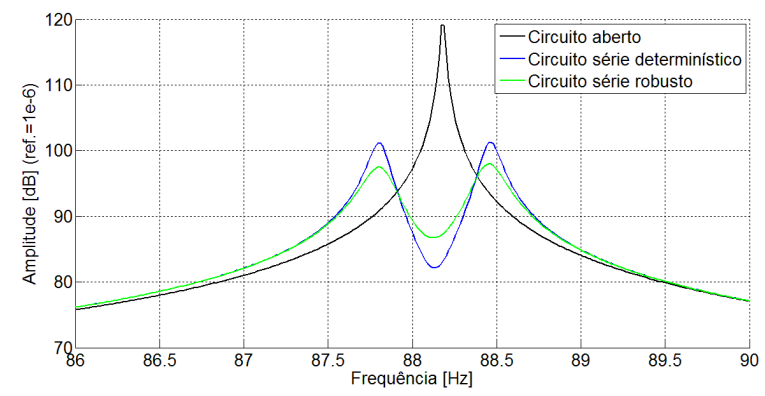

Figura 10. Amplitudes das FRFs para o primeiro modo - Circuito Série.

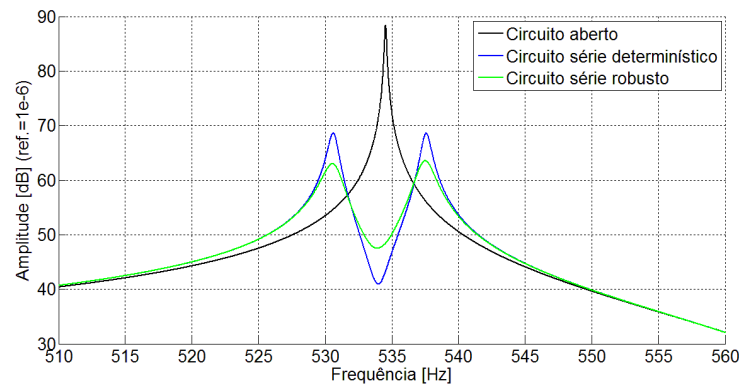

Figura 11. Amplitudes das FRFs para o segundo modo - Circuito Série. 


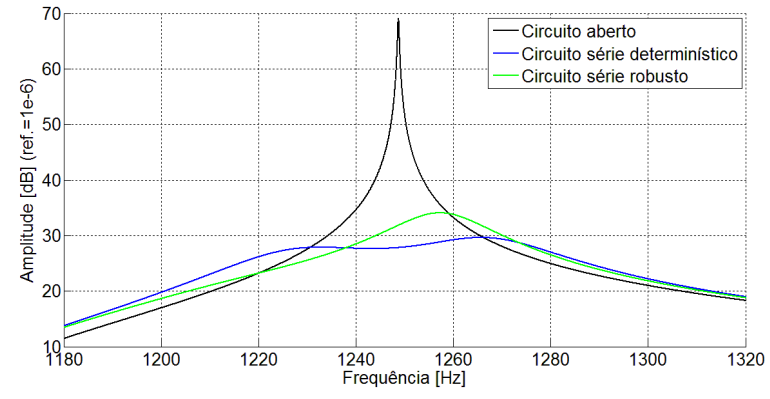

Figura 12. Amplitudes das FRFs para o terceiro modo - Circuito Série.
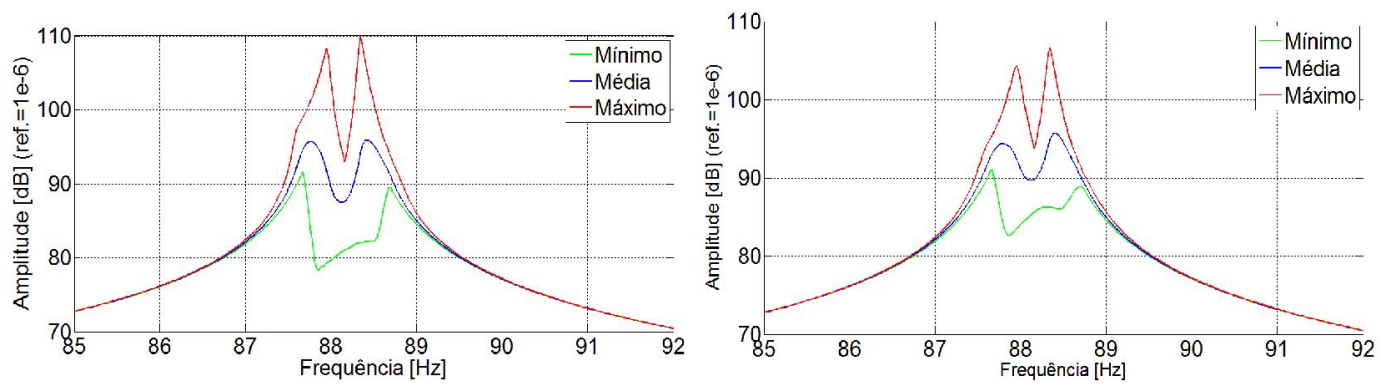

Figura 13. Envelopes de soluções para o primeiro modo - (a) Determinístico, (b) Robusto - Circuito Série.
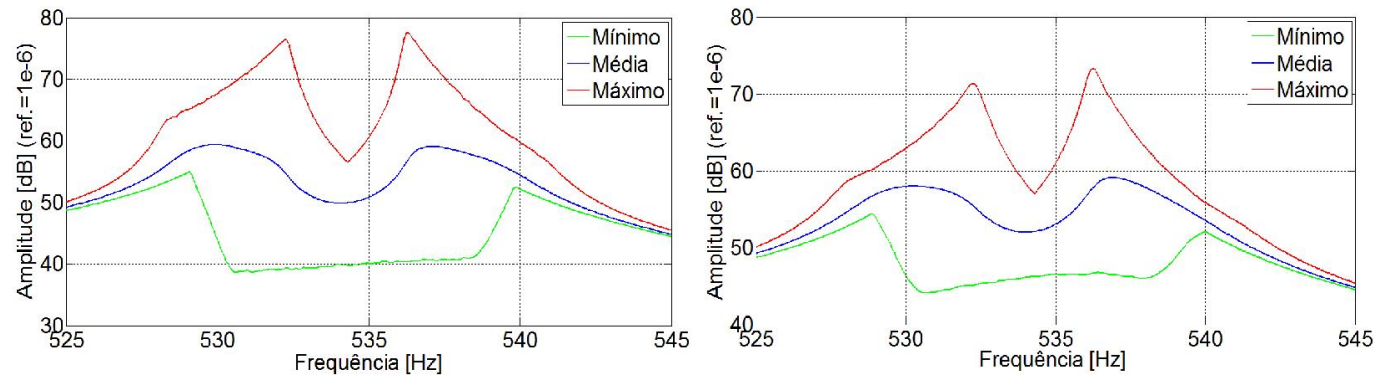

Figura 14. Envelopes de soluções para o Segundo modo - (a) Determinístico, (b) Robusto - Circuito Série.
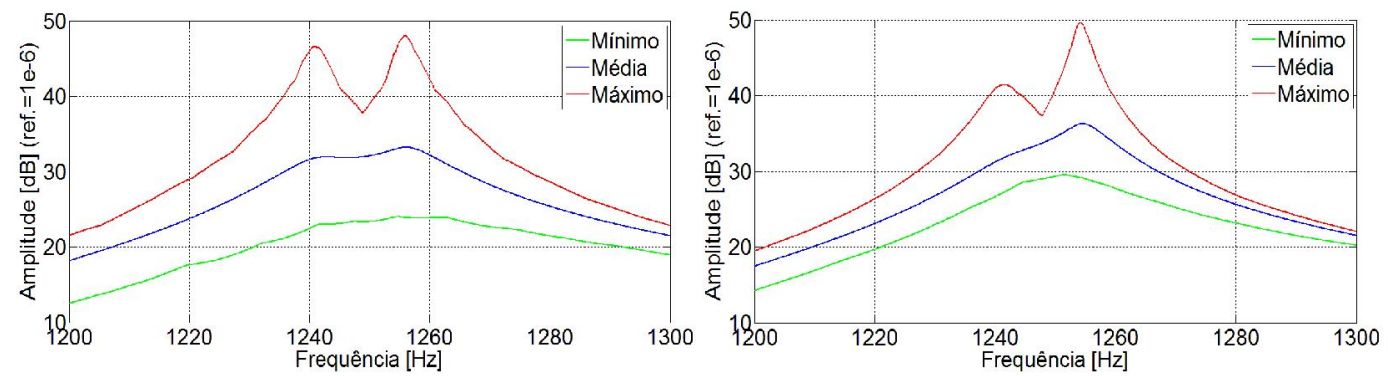

Figura 15. Envelopes de soluções para o terceiro modo - (a) Determinístico, (b) Robusto - Circuito Série.

\section{CONCLUSÕES}

Analisando os resultados mostrados nas Figs. 4 a 6 e nas Figs. 10 a 12, é possível observar que as soluções obtidas pela otimização determinística dominam as soluções robustas em termos de atenuação das amplitudes de vibração. Isso acontece em ambas as topologias dos circuitos e para todos os modos considerados na análise.

Considerando os envelopes de soluções apresentados nas Figs. 7 a 9 e nas Figs. 13 a 15, as soluções robustas obtidas em ambas as topologias de circuitos são mais robustas do que as soluções geradas na otimização determinística. Assim, fica evidenciada a importância em considerar incertezas paramétricas no procedimento de projeto de circuitos shunt multimodais.

Finalmente, comparando as duas topologias de circuitos shunt multimodais propostas a serem utilizadas para reduzir as vibrações de estruturas compostas, é possível concluir que o circuito com a topologia em série é mais 
eficiente, Entretanto, considerando a robustez, o circuito com topologia em paralelo é mais robusto do que o circuito com a topologia em série. Isto é consistente com o trabalho proposto por Wu (1996).

\section{AGRADECIMENTOS}

Os autores agradecem ao Conselho Nacional de Desenvolvimento Científico e Tecnológico - CNPq pelo apoio contínuo aos seus trabalhos de pesquisa, especialmente através dos projetos de pesquisa 308310/2010-1 (A.M.G. de Lima). Os autores expressam seus agradecimentos à FAPEMIG pelo suporte financeiro às atividades de pesquisa e ao INCT de Estruturas Inteligentes em Engenharia, juntamente fundado pelo CNPq, CAPES, FAPEMIG e PETROBRAS.

\section{REFERÊNCIAS}

Chee, C. Y. K. Static Shape Control of Laminated Composite Plate Smart Structure using Piezoelectric Actuators.2000. Pos-Doctorade Theses. - University of Sydney: Department of Aeronautical Engineering, Sydney, Austrália.

Faria, W. F. Modelagem por elementos finitos de placas compostas dotadas de sensores e atuadores piezelétricos: implementação computacional e avaliação numérica. 2006. 152f. Dissertação de Mestrado, Universidade Federal de Uberlândia, Uberlândia, MG.

Flaming, A.J., Behrens, S., Moheimani, S.O.R., Optimization and Implementation of Multimode Piezoelectric Shunt Damping Systems. IEEE/ASME Transactions on Mechatronics, Vol.7, no.1, March 2002.

Hagood, N.W., Von Flotow, V., 1991, "Damping of Structural Vibrations with Piezoelectric Materials and Passive Electrical Networks," Journal of Sound and Vibration, 146(2), pp. 243-268.

Hwang, W.; Hwang, W.; Park, H. C. Vibration Control of a Laminated Plate with Piezoelectric Sensor/Actuator: Finite Element Formulation and Modal Analysis. Journal of Intelligent Material Systems and Structures, v.4, n.4, p. 317329, 1994.

Nye, J. F. Physical Properties of Crystals: Their Representation by Tensors and Matrices. 4.ed. London: Oxford University Press, 1969.

Viana, F.C., 2005, “Amortecimento de Vibrações Usando Pastilhas Piezelétricas e Circuitos Shunt Passivos”, Dissertação de Mestrado, Universidade Federal de Uberlândia.

Wu, S. Y., "Piezoelectric shunts with parallel R-L circuit for structural damping and vibration control," inProc. SPIE Smart Structures and Materials, Passive Damping and Isolation, vol. 2720, SPIE, Mar. 1996, pp. 259-269.

Wu, S. Y., "Method for multiple mode shunt damping of structural vibration using a single PZT transducer," 1998.

Zambolini-Vicente, B.G.G.L., 2013, "Projeto Ótimo de Placas Compostas Incorporando Materiais Piezelétricos e Circuitos Elétricos Shunt para Controle Passivo de Vibrações”, Dissertação de Mestrado, Faculdade de Engenharia Mecânica, Universidade Federal de Uberlândia, Brasil.

\section{RESPONSABILIDADE AUTORAL}

“Os autores são os únicos responsáveis pelo conteúdo deste trabalho”. 
Congresso Nacional de Matemática Aplicada à Indústria, 18 a 21 de novembro de 2014, Caldas Novas - GO

\title{
OPTIMIZATION OF MULTIMODAL SHUNT CIRCUITS FOR THE PASSIVE CONTROL OF COMPOSITE STRUCTURES
}

\author{
Victor Augusto da Costa Silva, Victor_augusto_cn@hotmail.com \\ Bruno Gabriel Gustavo Leonardo Zambolini Vicente, Brunozv@gmail.com \\ Antônio Marcos Gonçalves de Lima, amglima@mecanica.ufu.br
}

Federal University of Uberlândia, School of Mechanical Engineering, Campus Sta Mônica, Uberlândia-MG, Brazil Federal University of Uberlândia, School of Mechanical Engineering, Campus Sta Mônica, Uberlândia-MG, Brazil Federal University of Uberlândia, School of Mechanical Engineering, Campus Sta Mônica, Uberlândia-MG, Brazil

\begin{abstract}
In the last decades, many efforts have been devoted to the modeling and design of engineering systems incorporating smart structures to the problem of vibration attenuation due to the combination of technological advances in the materials science and computers, and the numerical optimization techniques. Among the widely employed smart materials and structures it can be cited the use of piezoelectric materials to be combined with passive electrical shunt circuits. However, the most common strategy which has been employed is using shunt circuits that control only a single mode of vibration. Therefore, to control simultaneously a number of modes for an engineering structure, the strategy normally adopted is the use of various piezoelectric elements incorporated into the structure, where each piezoelectric is associated to a specific vibration mode circuit, causing the structure to certain problems such as the added weight. On the other hand, passive multimodal electrical circuits that control various vibration modes simultaneously by using only a single piezoelectric element present potential advantages. The effective design of multimodal shunt circuits as applied to real-word complex engineering structures can be conveniently carried-out by using modern robust multiobjective numerical optimization techniques which are capable of dealing with various objective and vulnerability functions, some of which can be conflicting. However, the large number of evaluations of the cost and vulnerability functions, combined with the typically high dimensions of finite element models of composite structures, makes robust optimizations very costly, sometimes unfeasible. Those difficulties motivate the study reported in this paper, in which a strategy is proposed consisting in the use of multiobjective evolutionary algorithms combined with metamodels. After discussing of the various theoretical aspects involved in the study, a numerical application is presented to illustrate the proposed robust optimization methodology for the robust-optimal design of multimodal shunt circuits applied to composite structures.
\end{abstract}

Keywords: Robust Optimization, Composite Structures, Multimodal Shunt Circuits, Passive Control, Uncertainties 